\title{
Master integrals for two-loop QCD corrections to quark quasi PDFs
}

\author{
Long-Bin Chen, ${ }^{a}$ Wei Wang ${ }^{b, 1}$ and Ruilin Zhu ${ }^{c, d, 2}$ \\ ${ }^{a}$ School of Physics and Materials Science, Guangzhou University, \\ Guangzhou 510006, China \\ ${ }^{b}$ INPAC, Shanghai Key Laboratory for Particle Physics and Cosmology, \\ MOE Key Lab for Particle Astrophysics and Cosmology, \\ School of Physics and Astronomy, Shanghai Jiao Tong University, \\ Shanghai, 200240, China \\ ${ }^{c}$ Department of Physics and Institute of Theoretical Physics, Nanjing Normal University, \\ Nanjing, Jiangsu 210023, China \\ ${ }^{d}$ Nuclear Science Division, Lawrence Berkeley National Laboratory, \\ Berkeley, CA 94720, U.S.A. \\ E-mail: chenlb@gzhu.edu.cn, wei.wang@sjtu.edu.cn, rlzhu@njnu.edu.cn
}

ABSTRACT: We compute the master integrals for the two-loop QCD corrections to quark quasi parton distribution functions (PDFs) in the large momentum effective theory (LaMET). With a proper canonical basis, we derive the analytical results for the three families of master integrals using the method of differential equations. The final expressions for the master integrals are given in terms of Goncharov polylogarithms. These results allow us to extract the two-loop short-distant matching coefficients between quark quasi and lightcone PDFs in LaMET, and are valuable to improve the determination of the nucleon PDFs from first principles in future.

KeYwords: Perturbative QCD, Lattice QCD

ARXIV EPRINT: 2006.10917

\footnotetext{
${ }^{1}$ Corresponding author.

${ }^{2}$ Corresponding author.
} 


\section{Contents}

1 Introduction 1

2 Notations and conventions $\quad 2$

3 The canonical basis $\quad 4$

4 Boundary conditions $\quad 9$

4.1 The rest boundary conditions for the integrals in the first family 11

4.2 The rest boundary conditions for the integrals in the second family 12

4.3 The rest boundary conditions for the integrals in the third family 12

5 Analytic results and validations $\quad \mathbf{1 2}$

$\begin{array}{lll}5.1 & \text { Results for off-shell quarks } & 12\end{array}$

$\begin{array}{lll}5.2 & \text { Results for on-shell quarks } & 14\end{array}$

$\begin{array}{lll}5.3 \text { Validations } & 15\end{array}$

$\begin{array}{llr}6 & \text { Discussions and conclusions } & 16\end{array}$

\section{Introduction}

In high energy physics, theoretical predictions for physical observables like cross sections are usually made under QCD factorization scheme, in which the scattering amplitude is split into a perturbative hard-kernel and low-energy matrix elements. The perturbative coefficient arises from the short-distant degrees of freedom, while the long-distant inputs, parton distribution functions (PDFs) and others, describe the longitudinal momentum distribution of unpolarized/polarized partons inside a hadron. The involved partons in the hadron move nearly at the speed of light. Thereby it is extremely difficult to directly calculate the PDFs from first-principles of QCD, i.e. Lattice QCD. Previous attempts in Lattice QCD make use of the operator product expansion and calculate the moments of PDFs. This approach was successful only for the lowest few moments of the lightcone PDFs [1], but studies of higher moments suffer from significantly large noises in the simulation. Thus a complete description of PDFs from first-principles is not established yet.

Recently a breakthrough to circumvent the above problem was proposed in refs. [2, 3], and is now formulated as the large momentum effective theory (LaMET). In this framework, instead of calculating the moments of lightcone PDFs one can explore the equal-time correlators, also called quasi observables, on the Lattice. The equal-time correlators have the same infrared structure with lightcone quantities, and more particularly under the large Lorentz boost, the quasi correlators approach the pertinent lightcone observables. Though 
they still have different ultraviolet behaviors, such differences are compensated by the perturbatively calculable coefficients. In the same spirit, other proposals like the "good lattice cross-section" $[4,5]$ and Ioffe-time "pseudo-distributions" [6] are also given in recent years.

Under the LaMET framework, tremendous progress has been made in recent years [764], and a lot of fairly good results are obtained, many of which are consistent with the phenomenological results extracted from the experiments [65] (see refs. [66, 67] for recent reviews). It is anticipated that with the increase of computing resources and the development of various techniques in future, Lattice simulations of quasi distributions will become more and more accurate. On the other hand, most of the available extractions of lightcone PDFs resort to the perturbative kernels at one-loop accuracy. Thus to further reduce theoretical uncertainties, the next-to-next-to-leading order (NNLO) predictions of the matching coefficients are inevitably required. In ref. [63], we have for the first time presented a NNLO calculation for the flavor non-diagonal contributions to the quark quasi distribution functions and explicitly validated the factorization hypothesis at NNLO accuracy. Many calculation details are not included in ref. [63].

Moreover the study of the mathematical properties of Feynman integrals has attracted increasing attention in the last decades, and significant progress was achieved in understanding the analytical behavior of multi-loop Feynman integrals. One of the most powerful tools to evaluate the master integrals analytically is the method of differential equations [68-72]. Along with the recent developments [73-76], this method has been widely applied to various processes. It has been pointed out in ref. [73] that in a generic multiloop calculation, a suitable basis (canonical basis) of master integrals can be chosen. In this canonical basis, the corresponding differential equations are greatly simplified, and their iterative solutions become straightforward in terms of the dimensional regularization parameter $\epsilon=\frac{4-D}{2}$. In addition, the determination of the boundary conditions is also greatly simplified.

In this work, we will present the detailed results for the two-loop Feynman integrals of QCD corrections to quark quasi PDFs. With the integration-by-parts (IBP) technique, all the two-loop QCD corrections to quark quasi PDFs are reduced into a set of integrals, called master integrals, which are then calculated using the method of differential equations. In section 2, we will set up the notations and conventions. Section 3 is devoted to the canonical basis of the master integrals, and section 4 details the boundary conditions for the integrals. Section 5 contains the analytical results for the master integrals and some validations through FIESTA are also given. A brief summary is given in the last section.

\section{Notations and conventions}

The quasi PDF for the quark $q_{i}$ in a hadron $H$ is defined as:

$$
\tilde{f}_{q_{i} / H}\left(y, \mu, P^{z}\right)=N \int \frac{d z}{4 \pi} e^{i z y P^{z}}\left\langle P\left|\bar{q}_{i}(z) \Gamma W(z, 0) q_{i}(0)\right| P\right\rangle,
$$

with $N$ being the normalization factor and $y \in[-\infty, \infty]$ being $\hat{z}$-component momentum fractions of the hadron carried by the parton. The $q_{i}$ is the quark field and the $W(z, 0)$ is 
the Wilson line from 0 to $z$ to maintain the gauge invariance. The $\Gamma$ is the Dirac matrix for the quasi PDF and two popular choices are $\Gamma=\gamma^{0}, \gamma^{z}$ for unpolarized PDFs. The gluon quasi PDFs can also be defined similarly.

Studying the quasi PDFs in perturbation theory requires the replacement of hadronic state by partonic state. Then one can calculate the perturbative contributions and extract the short-distant matching coefficients order by order. In the momentum space, the twoloop QCD corrections to quasi PDFs contain a momentum conservation in $z$ direction arising from the Fourier transformation as shown in eq. (2.1). This results in a delta function. In order to use the standard IBP technique, we employ the identity

$$
\delta\left(k_{z}-y p_{z}\right)=\frac{1}{2 \pi i}\left(\frac{1}{k_{z}-y p_{z}-i 0}-\frac{1}{k_{z}-y p_{z}+i 0}\right) .
$$

All the involved integrals in the two-loop contributions can be expressed by the three families of integrals, which are generically parameterized as

$$
\begin{aligned}
& I_{i_{1}, i_{2}, \ldots, i_{7}}^{1}=\int \mathcal{D}^{D} k_{1} \mathcal{D}^{D} k_{2} \frac{1}{\left(P_{1}+i 0\right)^{i_{1}}\left(P_{2}+i 0\right)^{i_{2}}\left(P_{3}+i 0\right)^{i_{3}}\left(P_{4}+i 0\right)^{i_{4}}\left(P_{5}+i 0\right)^{i_{5}}} \\
& \times \frac{1}{2 \pi i}\left(\frac{1}{\left(P_{6}+i 0\right)^{i_{6}}}-\frac{1}{\left(P_{6}-i 0\right)^{i_{6}}}\right) \frac{1}{2}\left(\frac{1}{\left(P_{7}+i 0\right)^{i_{7}}}+\frac{1}{\left(P_{7}-i 0\right)^{i_{7}}}\right),
\end{aligned}
$$

with

$$
\begin{array}{cl}
P_{1}=k_{1}^{2}, & P_{2}=k_{2}^{2}, \\
P_{3}=\left(k_{2}-p_{1}\right)^{2}, & P_{4}=\left(k_{1}+k_{2}\right)^{2}, \\
P_{5}=\left(k_{1}+k_{2}-p_{1}\right)^{2}, & P_{6}=n_{1} \cdot k_{1}+y p_{z}, \\
P_{7}=n_{1} \cdot k_{2} . & \\
I_{i_{1}, i_{2}, \ldots, i_{7}}^{2}=\int \mathcal{D}^{D} k_{1} \mathcal{D}^{D} k_{2} \frac{1}{\left(A_{1}+i 0\right)^{i_{1}}\left(A_{2}+i 0\right)^{i_{2}}\left(A_{3}+i 0\right)^{i_{3}}\left(A_{4}+i 0\right)^{i_{4}}\left(A_{5}+i 0\right)^{i_{5}}} \\
\times \frac{1}{2 \pi i}\left(\frac{1}{\left(A_{6}+i 0\right)^{i_{6}}}-\frac{1}{\left(A_{6}-i 0\right)^{i_{6}}}\right) \frac{1}{2}\left(\frac{1}{\left(A_{7}+i 0\right)^{i_{7}}}+\frac{1}{\left(A_{7}-i 0\right)^{i_{7}}}\right),
\end{array}
$$

with

$$
\begin{aligned}
& A_{1}=k_{1}^{2} \text {, } \\
& A_{3}=\left(k_{2}-p_{1}\right)^{2} \\
& A_{2}=k_{2}^{2} \\
& A_{5}=\left(k_{1}+k_{2}-p_{1}\right)^{2}, \\
& A_{4}=\left(k_{1}+k_{2}\right)^{2} \text {, } \\
& A_{7}=n_{1} \cdot k_{2} \text {. } \\
& I_{i_{1}, i_{2}, \ldots, i_{7}}^{3}=\int \mathcal{D}^{D} k_{1} \mathcal{D}^{D} k_{2} \frac{1}{\left(B_{1}+i 0\right)^{i_{1}}\left(B_{2}+i 0\right)^{i_{2}}\left(B_{3}+i 0\right)^{i_{3}}\left(B_{4}+i 0\right)^{i_{4}}\left(B_{7}+i 0\right)^{i_{7}}} \\
& \times \frac{1}{2 \pi i}\left(\frac{1}{\left(B_{6}+i 0\right)^{i_{6}}}-\frac{1}{\left(B_{6}-i 0\right)^{i_{6}}}\right) \frac{1}{2}\left(\frac{1}{\left(B_{5}+i 0\right)^{i_{5}}}+\frac{1}{\left(B_{5}-i 0\right)^{i_{5}}}\right),
\end{aligned}
$$


with

$$
\begin{array}{ll}
B_{1}=k_{1}^{2}, & B_{2}=k_{2}^{2}, \\
B_{3}=\left(k_{1}-p_{1}\right)^{2}, & B_{4}=\left(k_{2}+p_{1}\right)^{2}, \\
B_{5}=n_{1} \cdot\left(k_{2}-k_{1}+p_{1}\right), & B_{6}=n_{1} \cdot k_{1}+y p_{z}, \\
B_{7}=\left(k_{2}-k_{1}+p_{1}\right)^{2} . &
\end{array}
$$

The integration measure is defined as

$$
\mathcal{D}^{D} k_{i}=\frac{1}{i \pi^{D / 2} \mathrm{e}^{-\frac{4-D}{2} \gamma_{\mathrm{E}}}}\left(\frac{p_{z}^{2}}{\mu^{2}}\right)^{\left(\frac{4-D}{2}\right)} \mathrm{d}^{D} k_{i},
$$

and $D=4-2 \epsilon$. The momentum in above equations can be parameterized as

$$
p_{1}=\left(p_{0}, \overrightarrow{0}_{D-2}, p_{z}\right), n_{1}=\left(0, \overrightarrow{0}_{D-2}, 1\right)
$$

with $p_{1}^{2}=p_{1} \cdot p_{1}=p_{0}^{2}-p_{z}^{2}, n_{1}^{2}=-1$. When $p_{1}^{2} \leq 0$ (space-like and lightlike), all the integrals defined above are real.

After some algebraic manipulations and simplifications of the Feynman amplitudes, we encounter a number of tensor integrals. We will use FIRE packages [77-79] to perform the IBP reduction, after which all the integrals are reduced to the so-called master integrals. The first family of master integrals contains 36 linearly independent integrals, while the second and third family contain 32 and 28 integrals, respectively. To obtain the analytic results of master integrals, we will apply the method of differential equations and choose the canonical basis.

On the Lattice, the numerical simulation of quasi PDFs is performed in the discretized space-time, and to properly use the simulated results one needs to renormalize the bare quantities. A widely-adopted renormalization approach in the study of quasi PDFs is the so-called regularization-independent momentum subtraction scheme (RI/MOM) [80]. In this scheme, a counterterm with off-shell quarks $p_{1}^{2}=-\mu_{R}^{2}<0$ is introduced. Thus to match the RI/MOM results from Lattice QCD onto the $\overline{\mathrm{MS}} \mathrm{PDFs}$ in the continuum theory, one needs to calculate the two-loop diagrams in both cases with $p_{1}^{2}=0$ and $p_{1}^{2}<0$.

\section{The canonical basis}

In this section, we show the canonical basis for three families of master integrals. To rationalize the squared root $\sqrt{p_{1}^{2}+p_{z}^{2}}$ that appears in the differential equations and canonical basis, we define a dimensionless parameter

$$
z=\frac{\sqrt{p_{1}^{2}+p_{z}^{2}}}{p_{z}}=\frac{p_{0}}{p_{z}} .
$$

After the IBP reduction, we obtain 36 independent master integrals for the first family of integrals. Following the strategy proposed by Henn [73], we construct the canonical basis, 
and the vector of the canonical basis $\mathbf{g}^{1}$ is built up with 36 functions $g_{i}^{1}(y, z, \epsilon)(i=1 \ldots 36)$, defined in terms of the linear combinations of 36 master integrals:

$$
\begin{aligned}
& g_{1}^{1}=\epsilon(y+1) p_{z} I_{0,0,2,2,0,1,0}^{1}, \\
& g_{2}^{1}=\epsilon y p_{z} I_{0,2,0,2,0,1,0}^{1} \text {, } \\
& g_{3}^{1}=\epsilon(y-1) p_{z} I_{0,2,0,0,2,1,0}^{1}, \\
& g_{4}^{1}=\epsilon y p_{z} p_{1}^{2} I_{2,2,1,0,0,1,0}^{1} \text {, } \\
& g_{5}^{1}=\epsilon^{2} \sqrt{p_{1}^{2}+p_{z}^{2}} I_{0,1,1,0,2,1,0}^{1}, \\
& g_{6}^{1}=\epsilon\left(p_{1}^{2}-4 y(y-1) p_{z}^{2}\right) I_{0,1,1,0,2,2,0}^{1}+8 \epsilon^{2}(2 y-1) p_{z} I_{0,1,1,0,2,1,0}^{1} \\
& +\epsilon(y-1) p_{z} I_{0,2,0,0,2,1,0}^{1}+\epsilon y p_{z} I_{0,2,0,2,0,1,0}^{1}, \\
& g_{7}^{1}=\epsilon^{2} \sqrt{p_{1}^{2}+p_{z}^{2}} I_{1,1,0,0,2,1,0}^{1}, \\
& g_{8}^{1}=\epsilon\left(p_{1}^{2}-4 y(y-1) p_{z}^{2}\right) I_{1,1,0,0,2,2,0}^{1}+6 \epsilon^{2}(2 y-1) p_{z} I_{1,1,0,0,2,1,0}^{1}, \\
& g_{9}^{1}=\epsilon^{2} \sqrt{p_{1}^{2}+p_{z}^{2}} I_{0,1,1,2,0,1,0}^{1}, \\
& g_{10}^{1}=\epsilon\left(p_{1}^{2}-4 y(y+1) p_{z}^{2}\right) I_{0,1,1,2,0,2,0}^{1}+8 \epsilon^{2}(2 y+1) p_{z} I_{0,1,1,2,0,1,0}^{1} \\
& +\epsilon(y+1) p_{z} I_{0,0,2,2,0,1,0}^{1}+\epsilon y p_{z} I_{0,2,0,2,0,1,0}^{1}, \\
& g_{11}^{1}=\epsilon^{2} \sqrt{p_{1}^{2}+p_{z}^{2}} I_{1,0,2,1,0,1,0}^{1}, \\
& g_{12}^{1}=\epsilon\left(p_{1}^{2}-4 y(y+1) p_{z}^{2}\right) I_{1,0,2,1,0,2,0}^{1}+6 \epsilon^{2}(2 y+1) p_{z} I_{1,0,2,1,0,1,0}^{1} \text {, } \\
& g_{13}^{1}=\epsilon^{3} \sqrt{p_{1}^{2}+p_{z}^{2}} I_{1,1,1,0,1,1,0}^{1}, \\
& g_{14}^{1}=\epsilon^{3} \sqrt{p_{1}^{2}+p_{z}^{2}} I_{1,1,1,1,0,1,0}^{1}, \\
& g_{15}^{1}=\epsilon^{2}\left(p_{1}^{2}+p_{z}^{2}\right) I_{0,1,1,1,1,2,0}^{1}, \\
& g_{16}^{1}=\epsilon^{2} \sqrt{p_{1}^{2}+p_{z}^{2}}\left(y p_{z} I_{0,1,1,1,1,2,0}^{1}+(1-4 \epsilon) I_{0,1,1,1,1,1,0}^{1}\right) \text {, } \\
& g_{17}^{1}=\epsilon^{2} \sqrt{p_{1}^{2}+p_{z}^{2}}\left(\frac{p_{z}}{2} I_{0,1,1,1,1,2,0}^{1}+p_{1}^{2} I_{0,2,1,1,1,1,0}^{1}\right) \text {, } \\
& g_{18}^{1}=\epsilon y(y-1) p_{z}^{2} I_{2,0,0,0,2,1,1}^{1} \text {, } \\
& g_{19}^{1}=\epsilon y^{2} p_{z}^{2} I_{2,0,0,2,0,1,1}^{1} \text {, } \\
& g_{20}^{1}=\epsilon y p_{z}^{2} I_{2,0,2,0,0,1,1}^{1} \text {, } \\
& g_{21}^{1}=\epsilon p_{z} I_{0,0,2,0,1,2,1}^{1}, \\
& g_{22}^{1}=\epsilon p_{z} I_{0,0,2,1,0,2,1}^{1} \text {, } \\
& g_{23}^{1}=\epsilon^{2} y p_{z} \sqrt{p_{1}^{2}+p_{z}^{2}} I_{0,1,1,2,0,1,1}^{1} \text {, } \\
& g_{24}^{1}=\epsilon^{2} \sqrt{p_{1}^{2}+p_{z}^{2}} I_{1,0,1,1,0,2,1}^{1}, \\
& g_{25}^{1}=\epsilon^{2} p_{z} \sqrt{p_{1}^{2}+p_{z}^{2}} I_{1,0,2,1,0,1,1}^{1} \text {, } \\
& g_{26}^{1}=\epsilon^{2} y p_{z} \sqrt{p_{1}^{2}+p_{z}^{2}} I_{2,0,1,1,0,1,1}^{1} \text {, } \\
& g_{27}^{1}=\epsilon^{2}\left(y p_{z} I_{1,0,1,1,0,2,1}^{1}-2 p_{z} I_{1,0,2,1,0,1,0}^{1}-2 p_{z}^{2} I_{1,0,2,1,0,1,1}^{1}+(1-6 \epsilon) I_{1,0,1,1,0,1,1}^{1}\right) \text {, }
\end{aligned}
$$




$$
\begin{aligned}
& g_{28}^{1}=\epsilon^{2} \sqrt{p_{1}^{2}+p_{z}^{2}} I_{1,1,0,0,1,1,2}^{1}, \\
& g_{29}^{1}=\epsilon^{2} \sqrt{p_{1}^{2}+p_{z}^{2}} I_{1,1,0,0,1,2,1}^{1}, \\
& g_{30}^{1}=\epsilon^{2}\left(p_{z} y I_{1,1,0,0,1,2,1}^{1}-p_{z} I_{1,1,0,0,1,1,2}^{1}+2 p_{z} I_{1,1,0,0,2,1,0}^{1}+(1-6 \epsilon) I_{1,1,0,0,1,1,1}^{1}\right), \\
& g_{31}^{1}=\epsilon^{2} \sqrt{p_{1}^{2}+p_{z}^{2}} I_{1,1,1,0,0,2,1}^{1}, \\
& g_{32}^{1}=\epsilon^{2}(1-2 \epsilon) \frac{\sqrt{p_{1}^{2}+p_{z}^{2}}}{p_{z} y} I_{1,0,0,1,1,1,1}^{1}, \\
& g_{33}^{1}=\epsilon^{2} \sqrt{p_{1}^{2}+p_{z}^{2}} I_{0,1,1,0,1,2,1}^{1}, \\
& g_{34}^{1}=\epsilon^{2} p_{z} \sqrt{p_{1}^{2}+p_{z}^{2}} I_{0,0,2,1,1,1,1}^{1}, \\
& g_{35}^{1}=\epsilon^{3}\left(p_{1}^{2}+p_{z}^{2}\right) I_{0,1,1,1,1,1,1}^{1}, \\
& g_{36}^{1}=\epsilon^{3} p_{1}^{2} y p_{z} \sqrt{p_{1}^{2}+p_{z}^{2}} I_{1,1,1,1,1,1,1}^{1} .
\end{aligned}
$$

The differential equations for the canonical basis in the first family can then be obtained directly as

$$
\mathrm{d} \mathbf{g}^{1}(y, z ; \epsilon)=\epsilon \mathrm{d} \tilde{\mathrm{M}}(y, z) \mathbf{g}^{1}(y, z ; \epsilon)
$$

with

$$
\begin{aligned}
\tilde{\mathrm{M}}(y, z)= & \mathrm{M}_{1} \ln (z)+\mathrm{M}_{2} \ln (z-1)+\mathrm{M}_{3} \ln (z+1)+\mathrm{M}_{4} \ln (z-2 y+1) \\
& +\mathrm{M}_{5} \ln (z+2 y-1)+\mathrm{M}_{6} \ln (z-2 y-1)+\mathrm{M}_{7} \ln (z+2 y+1) \\
& +\mathrm{M}_{8} \ln (z-y)+\mathrm{M}_{9} \ln (z+y)+\mathrm{M}_{10} \ln (y-1)+\mathrm{M}_{11} \ln (y)+\mathrm{M}_{12} \ln (y+1) .
\end{aligned}
$$

The $\mathrm{M}_{i}$ are $36 \times 36$ rational matrices whose explicit forms are given in the Supplementary material of this manuscript.

The canonical basis $g_{i}^{2}(y, z, \epsilon)(i=1 \ldots 32)$ for the second family is similarly chosen as:

$$
\begin{aligned}
& g_{1}^{2}=\epsilon y p_{z} I_{2,2,0,0,0,1,0}^{2}, \\
& g_{2}^{2}=\epsilon(y-1) p_{z} I_{2,0,2,0,0,1,0}^{2}, \\
& g_{3}^{2}=\epsilon(y-1) p_{z}^{2} I_{0,0,2,0,2,1,1}^{2} \\
& g_{4}^{2}=\epsilon y p_{z}^{2} I_{0,0,2,2,0,1,1}^{2} \\
& g_{5}^{2}=\epsilon(y-1) p_{z} p_{1}^{2} I_{0,2,1,0,2,1,0}^{2}, \\
& g_{6}^{2}=\epsilon y p_{z} p_{1}^{2} I_{0,2,1,2,0,1,0}^{2} \\
& g_{7}^{2}=\epsilon y(y-1) p_{z}^{2} I_{2,0,0,0,2,1,1}^{2} \\
& g_{8}^{2}=\epsilon y^{2} p_{z}^{2} I_{2,0,0,2,0,1,1}^{2} \\
& g_{9}^{2}=\epsilon p_{z} I_{1,0,2,0,0,2,1}^{2} \\
& g_{10}^{2}=\epsilon \sqrt{p_{1}^{2}+p_{z}^{2}} I_{1,0,2,1,0,1,0}^{2}, \\
& g_{11}^{2}=\epsilon\left(p_{1}^{2}-4 y(y-1) p_{z}^{2}\right) I_{1,0,2,1,0,2,0}^{2}+6 \epsilon^{2}(2 y-1) p_{z} I_{1,0,2,1,0,1,0}^{2}, \\
& g_{12}^{2}=\epsilon^{2} \sqrt{p_{1}^{2}+p_{z}^{2}} I_{1,2,0,0,1,1,0}^{2},
\end{aligned}
$$




$$
\begin{aligned}
g_{13}^{2}= & \epsilon\left(p_{1}^{2}-4 y(y-1) p_{z}^{2}\right) I_{1,2,0,0,1,2,0}^{2}+6 \epsilon^{2}(2 y-1) p_{z} I_{1,2,0,0,1,1,0}^{2} \\
g_{14}^{2}= & \epsilon^{2} \sqrt{p_{1}^{2}+p_{z}^{2}} I_{2,1,1,0,0,1,0}^{2} \\
g_{15}^{2}= & \epsilon\left(p_{1}^{2}-4 y(y-1) p_{z}^{2}\right) I_{2,1,1,0,0,2,0}^{2}+8 \epsilon^{2}(2 y-1) p_{z} I_{2,1,1,0,0,1,0}^{2} \\
& +\epsilon y p_{z} I_{2,2,0,0,0,1,0}^{2}+\epsilon(y-1) p_{z} I_{2,0,2,0,0,1,0}^{2} \\
g_{16}^{2}= & \epsilon^{2} \sqrt{p_{1}^{2}+p_{z}^{2}} I_{1,1,0,0,1,2,1}^{2} \\
g_{17}^{2}= & \epsilon^{2} y p_{z} \sqrt{p_{1}^{2}+p_{z}^{2}} I_{2,1,0,0,1,1,1}^{2} \\
g_{18}^{2}= & \epsilon^{2}\left((1-6 \epsilon) I_{1,1,0,0,1,1,1}^{2}+(y-1) p_{z} I_{1,1,0,0,1,2,1}^{2}-2 p_{z} I_{1,2,0,0,1,1,0}^{2}-2 y p_{z}^{2} I_{2,1,0,0,1,1,1}^{2}\right) \\
g_{19}^{2}= & \epsilon^{2} \sqrt{p_{1}^{2}+p_{z}^{2}} I_{1,0,1,1,0,2,1}^{2} \\
g_{20}^{2}= & \epsilon^{2} y p_{z} \sqrt{p_{1}^{2}+p_{z}^{2}} I_{1,0,1,2,0,1,1}^{2} \\
g_{21}^{2}= & \epsilon^{2} p_{z} \sqrt{p_{1}^{2}+p_{z}^{2}} I_{1,0,2,1,0,1,1}^{2} \\
g_{22}^{2}= & \epsilon^{2}\left((1-6 \epsilon) I_{1,0,1,1,0,1,1}^{2}+y p_{z} I_{1,0,1,1,0,2,1}^{2}-2 p_{z}^{2} I_{1,0,2,1,0,1,1}^{2}-2 p_{z} I_{1,0,2,1,0,1,0}^{2}\right) \\
g_{23}^{2}= & \epsilon^{2} y p_{z} \sqrt{p_{1}^{2}+p_{z}^{2}} I_{2,1,1,0,0,1,1}^{2} \\
g_{24}^{2}= & \epsilon^{2}(y-1) p_{z} \sqrt{p_{1}^{2}+p_{z}^{2}} I_{0,1,1,0,2,1,1}^{2} \\
g_{25}^{2}= & \epsilon^{2}(y-1) p_{z} \sqrt{p_{1}^{2}+p_{z}^{2}} I_{1,1,1,0,1,2,0}^{2} \\
g_{26}^{2}= & \epsilon^{2} y p_{z} \sqrt{p_{1}^{2}+p_{z}^{2}} I_{2,0,0,1,1,1,1}^{2} \\
g_{27}^{2}= & \epsilon^{2} y p_{z} \sqrt{p_{1}^{2}+p_{z}^{2}} I_{1,1,1,1,0,2,0}^{2} \\
g_{28}^{2}= & \epsilon^{2} y p_{z} \sqrt{p_{1}^{2}+p_{z}^{2}} I_{0,1,1,2,0,1,1}^{2} \\
g_{29}^{2}= & \epsilon^{2} p_{1}^{2} \sqrt{p_{1}^{2}+p_{z}^{2}} I_{0,2,1,1,1,1,0}^{2} \\
g_{30}^{2}= & \epsilon^{2} p_{z} \sqrt{p_{1}^{2}+p_{z}^{2}} I_{0,0,2,1,1,1,1}^{2} \\
g_{31}^{2}= & \epsilon^{3}\left(p_{1}^{2}+p_{z}^{2}\right) I_{0,1,1,1,1,1,1}^{2} \\
g_{32}^{2}= & \epsilon^{3} y p_{1}^{2} p_{z} \sqrt{p_{1}^{2}+p_{z}^{2}} I_{1,1,1,1,1,1,1}^{2} \\
& \\
&
\end{aligned}
$$

The differential equations for above canonical basis can be expressed as

$$
\mathrm{d} \mathbf{g}^{2}(y, z ; \epsilon)=\epsilon \mathrm{d} \tilde{\mathrm{N}}(y, z) \mathbf{g}^{2}(y, z ; \epsilon)
$$

with

$$
\begin{aligned}
\tilde{\mathrm{N}}(y, z)= & \mathrm{N}_{1} \ln (z)+\mathrm{N}_{2} \ln (z-1)+\mathrm{N}_{3} \ln (z+1)+\mathrm{N}_{4} \ln (z-2 y+1)+\mathrm{N}_{5} \ln (z+2 y-1) \\
& +\mathrm{N}_{6} \ln (z-2 y-1)+\mathrm{N}_{7} \ln (z+2 y+1)+\mathrm{N}_{8} \ln (y-1)+\mathrm{N}_{9} \ln (y),
\end{aligned}
$$

where $\mathrm{N}_{i}$ are $32 \times 32$ rational matrices. 
For the third family of integrals, the canonical basis $g_{i}^{3}(y, z, \epsilon)(i=1 \ldots 28)$ is chosen as:

$$
\begin{aligned}
& g_{1}^{3}=\epsilon y(1-y) p_{z}^{2} I_{0,0,2,2,1,1,0}^{3}, \\
& g_{2}^{3}=\epsilon(1-y)^{2} p_{z}^{2} I_{0,2,2,0,1,1,0}^{3}, \\
& g_{3}^{3}=\epsilon(y-1) p_{1}^{2} p_{z} I_{0,2,2,1,0,1,0}^{3}, \\
& g_{4}^{3}=\epsilon y^{2} p_{z}^{2} I_{2,0,0,2,1,1,0}^{3} \text {, } \\
& g_{5}^{3}=\epsilon y(1-y) p_{z}^{2} I_{2,2,0,0,1,1,0}^{3}, \\
& g_{6}^{3}=\epsilon y p_{1}^{2} p_{z} I_{2,2,0,1,0,1,0}^{3} \text {, } \\
& g_{7}^{3}=\epsilon^{2} \frac{(1-2 \epsilon) \sqrt{p_{1}^{2}+p_{z}^{2}}}{(1-y) p_{z}} I_{0,1,1,1,1,1,0}^{3}, \\
& g_{8}^{3}=\epsilon^{2} \frac{(1-2 \epsilon) \sqrt{p_{1}^{2}+p_{z}^{2}}}{y p_{z}} I_{1,0,1,1,1,1,0}^{3}, \\
& g_{9}^{3}=\epsilon^{2} \frac{(1-2 \epsilon) \sqrt{p_{1}^{2}+p_{z}^{2}}}{y p_{z}} I_{1,1,0,1,1,1,0}^{3}, \\
& g_{10}^{3}=\epsilon^{2} \frac{(1-2 \epsilon) \sqrt{p_{1}^{2}+p_{z}^{2}}}{(1-y) p_{z}} I_{1,1,1,0,1,1,0}^{3}, \\
& g_{11}^{3}=\epsilon^{2}(1-2 \epsilon) \sqrt{p_{1}^{2}+p_{z}^{2}} I_{1,1,1,1,0,1,0}^{3}, \\
& g_{12}^{3}=\epsilon^{3}\left(p_{1}^{2}+p_{z}^{2}\right) I_{1,1,1,1,1,1,0}^{3}, \\
& g_{13}^{3}=\operatorname{\epsilon yp}_{z} I_{0,0,0,2,0,1,2}^{3} \text {, } \\
& g_{14}^{3}=\epsilon(y-1) p_{z} I_{0,2,0,0,0,1,2}^{3} \text {, } \\
& g_{15}^{3}=\epsilon^{2} \sqrt{p_{1}^{2}+p_{z}^{2}} I_{0,0,1,2,0,1,1}^{3}, \\
& g_{16}^{3}=\epsilon\left(p_{1}^{2}-4 y(y-1) p_{z}^{2}\right) I_{0,0,1,2,0,2,1}^{3}+6 \epsilon^{2}(2 y-1) p_{z} I_{0,0,1,2,0,1,1}^{3}, \\
& g_{17}^{3}=\epsilon^{2} \sqrt{p_{1}^{2}+p_{z}^{2}} I_{0,1,0,1,0,1,2}^{3} \text {, } \\
& g_{18}^{3}=\epsilon\left(p_{1}^{2}-4 y(y-1) p_{z}^{2}\right) I_{0,1,0,1,0,2,2}^{3}+8 \epsilon^{2}(2 y-1) p_{z} I_{0,1,0,1,0,1,2}^{3} \\
& +\epsilon y I_{0,0,0,2,0,1,2}^{3}+\epsilon(y-1) I_{0,2,0,0,0,1,2}^{3}, \\
& g_{19}^{3}=\epsilon^{2} \sqrt{p_{1}^{2}+p_{z}^{2}} I_{1,2,0,0,0,1,1}^{3}, \\
& g_{20}^{3}=\epsilon\left(p_{1}^{2}-4 y(y-1) p_{z}^{2}\right) I_{1,2,0,0,0,2,1}^{3}+6 \epsilon^{2}(2 y-1) p_{z} I_{1,2,0,0,0,1,1}^{3} \text {, } \\
& g_{21}^{3}=\epsilon^{2} \sqrt{p_{1}^{2}+p_{z}^{2}} I_{0,1,1,1,0,1,1}^{3} \text {, } \\
& g_{22}^{3}=\epsilon^{2} \sqrt{p_{1}^{2}+p_{z}^{2}} I_{1,1,0,0,2,1,1}^{3} \text {, } \\
& g_{23}^{3}=\epsilon^{2} \sqrt{p_{1}^{2}+p_{z}^{2}} I_{1,1,0,0,1,2,1}^{3} \text {, } \\
& g_{24}^{3}=\epsilon^{2}\left((1-6 \epsilon) I_{1,1,0,0,1,1,1}^{3}+y p_{z} I_{1,1,0,0,1,2,1}^{3}-p_{z} I_{1,1,0,0,2,1,1}^{3}+2 p_{z} I_{1,2,0,0,0,1,1}^{3}\right) \text {, } \\
& g_{25}^{3}=\epsilon^{2} y p_{z} \sqrt{p_{1}^{2}+p_{z}^{2}} I_{0,0,1,2,1,1,1}^{3},
\end{aligned}
$$




$$
\begin{aligned}
& g_{26}^{3}=\epsilon^{2} \sqrt{p_{1}^{2}+p_{z}^{2}} I_{0,0,1,1,1,2,1}^{3}, \\
& g_{27}^{3}=\epsilon^{2}\left((1-6 \epsilon) I_{0,0,1,1,1,1,1}^{3}+(y-1) p_{z} I_{0,0,1,1,1,2,1}^{3}+2 p_{z} I_{0,0,1,2,0,1,1}^{3}-2 y p_{z}^{2} I_{0,0,1,2,1,1,1}^{3}\right), \\
& g_{28}^{3}=\epsilon^{3} \sqrt{p_{1}^{2}+p_{z}^{2}} I_{1,1,0,1,0,1,1}^{3} .
\end{aligned}
$$

The corresponding differential equations of canonical basis in the third family can be formulated as

$$
\mathrm{d} \mathbf{g}^{3}(y, z ; \epsilon)=\epsilon \mathrm{d} \tilde{\mathrm{L}}(y, z) \mathbf{g}^{3}(y, z ; \epsilon)
$$

with

$$
\begin{aligned}
\tilde{\mathrm{L}}(y, z)= & \mathrm{L}_{1} \ln (z)+\mathrm{L}_{2} \ln (z-1)+\mathrm{L}_{3} \ln (z+1)+\mathrm{L}_{4} \ln (z-2 y+1) \\
& +\mathrm{L}_{5} \ln (z+2 y-1)+\mathrm{L}_{6} \ln (y-1)+\mathrm{L}_{7} \ln (y)
\end{aligned}
$$

where $\mathrm{L}_{i}$ are $28 \times 28$ rational matrices.

All the rational matrices $\left(\mathrm{M}_{i}, \mathrm{~N}_{i}, \mathrm{~L}_{i}\right)$ as well as the electronic form of the canonical basis are presented in the Supplementary material.

Although there are three families of master integrals for the NNLO corrections to quark quasi PDFs, some integrals in different families are related. After some inspection, we find the following relations:

$$
\begin{aligned}
& g_{2}^{1}=g_{1}^{2}=g_{13}^{3}, g_{3}^{1}=g_{2}^{2}=g_{14}^{3}, g_{19}^{1}=-g_{8}^{2}=g_{4}^{3}, \\
& g_{7}^{1}=g_{10}^{2}=g_{19}^{3}, g_{8}^{1}=g_{11}^{2}=g_{20}^{3}, g_{5}^{1}=g_{14}^{2}=g_{17}^{3}, \\
& g_{6}^{1}=g_{15}^{2}=g_{18}^{3} \\
& g_{31}^{1}=-2 g_{28}^{2}, g_{18}^{1}=-g_{5}^{3}, g_{28}^{1}=g_{22}^{3}, g_{29}^{1}=g_{23}^{3}, g_{30}^{1}=g_{24}^{3}, \\
& g_{7}^{2}=g_{1}^{3}, g_{5}^{2}=g_{3}^{3}, g_{6}^{2}=g_{6}^{3}, g_{12}^{2}=g_{15}^{3}, g_{13}^{2}=g_{16}^{3}, \\
& g_{25}^{2}=g_{21}^{3}, g_{17}^{2}=-g_{25}^{3}, g_{16}^{2}=-g_{26}^{3}, g_{18}^{2}=-g_{27}^{3}, \\
& g_{8}^{3}=-2 g_{26}^{2}, g_{11}^{3}=-g_{29}^{2}, g_{28}^{3}=\left(g_{27}^{2}+g_{10}^{2}-g_{14}^{2}\right) / 2 .
\end{aligned}
$$

\section{Boundary conditions}

In order to obtain the analytic results for the basis given in the last section, one needs to determine the boundary conditions first. In this section we present the determination of these boundary conditions.

We find that the analytic results for

$$
\begin{aligned}
& \left\{g_{1}^{1}, g_{2}^{1}, g_{3}^{1}, g_{4}^{1}, g_{18}^{1}, g_{19}^{1}, g_{20}^{1}\right\}, \\
& \left\{g_{1}^{2}, g_{2}^{2}, g_{3}^{2}, g_{4}^{2}, g_{5}^{2}, g_{6}^{2}, g_{7}^{2}, g_{8}^{2}\right\}, \\
& \left\{g_{1}^{3}, g_{2}^{3}, g_{3}^{3}, g_{4}^{3}, g_{5}^{3}, g_{6}^{3}, g_{13}^{3}, g_{14}^{3}\right\},
\end{aligned}
$$


can be obtained by directly performing the integration. For the reader's convenience, we show the results for the $g_{1}^{1}, g_{2}^{1}, g_{3}^{1}$ as follows:

$$
\begin{aligned}
g_{1}^{1}= & \operatorname{Sgn}(y+1)\left(-2+\epsilon\left[4 \ln \left(4(y+1)^{2}\right)\right]-\frac{1}{3} \epsilon^{2}\left[12 \ln \left((y+1)^{2}\right) \ln \left(16(y+1)^{2}\right)\right.\right. \\
& \left.\left.+5 \pi^{2}+12 \ln ^{2}(4)\right]+\mathcal{O}\left(\epsilon^{3}\right)\right), \\
g_{2}^{1}= & \operatorname{Sgn}(y)\left(-2+\epsilon\left[4 \ln \left(4 y^{2}\right)\right]-\frac{1}{3} \epsilon^{2}\left[12 \ln \left(y^{2}\right) \ln \left(16 y^{2}\right)+5 \pi^{2}+12 \ln ^{2}(4)\right]+\mathcal{O}\left(\epsilon^{3}\right)\right), \\
g_{3}^{1}= & \operatorname{Sgn}(y-1)\left(-2+\epsilon\left[4 \ln \left(4(y-1)^{2}\right)\right]-\frac{1}{3} \epsilon^{2}\left[12 \ln \left((y-1)^{2}\right) \ln \left(16(y-1)^{2}\right)\right.\right. \\
& \left.\left.+5 \pi^{2}+12 \ln ^{2}(4)\right]+\mathcal{O}\left(\epsilon^{3}\right)\right) .
\end{aligned}
$$

The above results are valid in the entire range of $y$ : $(-\infty<y<\infty)$.

Based on the one-loop results for the matching coefficients for the quark quasi PDFs $[14,59]$, we notice that in the $(0<y<1)$ region (the so-called physical region which overlaps with the kinematic region for the lightcone PDFs), the analytic results have logarithmic divergences at $p_{1}^{2}=0(z=1)$, but are regular at $p_{1}^{2}=4 y(y-1) p_{z}^{2}(z=2 y-1)$. In the $(y<0, y>1)$ regions the perturbative results are regular at $p_{1}^{2}=0(z=1)$, but are singular at $p_{1}^{2}=4 y(y-1) p_{z}^{2}(z=2 y-1)$. It is also interesting to notice that in the space-like region with $p_{1}^{2}=-p_{z}^{2}(z=0)$, the one-loop integrals are also regular. Though the two-loop integrals are much more complicated, we expect these analyticity properties also hold which will be used to determine the boundary conditions for two-loop integrals. The analytic results obtained with this assumption have been validated in the comparison with the results from FIESTA.

For illustration, we consider $g_{7}^{1}$ and $g_{8}^{1}$, whose differential equations are

$$
\begin{aligned}
& \frac{\partial g_{7}^{1}}{\partial z}=\frac{\epsilon}{4}\left(\frac{8 g_{7}^{1}}{z}-\frac{6 g_{7}^{1}-g_{8}^{1}}{z-2 y+1}-\frac{6 g_{7}^{1}+g_{8}^{1}}{z+2 y-1}+\frac{2 g_{3}^{1}-6 g_{7}^{1}+g_{8}^{1}}{z-1}-\frac{2 g_{3}^{1}+6 g_{7}^{1}+g_{8}^{1}}{z+1}\right), \\
& \frac{\partial g_{8}^{1}}{\partial z}=\frac{\epsilon}{2}\left(\frac{6 g_{7}^{1}-g_{8}^{1}}{z-2 y+1}-\frac{6 g_{7}^{1}+g_{8}^{1}}{z+2 y-1}+\frac{-2 g_{3}^{1}+6 g_{7}^{1}-g_{8}^{1}}{z-1}-\frac{2 g_{3}^{1}+6 g_{7}^{1}+g_{8}^{1}}{z+1}\right) .
\end{aligned}
$$

Since all integrals do not have any singularity at $p_{1}^{2}=-p_{z}^{2}(z=0)$, we find that the above equations imply $g_{7}^{1}=0$ at $p_{1}^{2}=-p_{z}^{2}(z=0)$. In the physical region $(0<y<1)$, the integrals $\left(g_{7}^{1}, g_{8}^{1}\right)$ are singular at $p_{1}^{2}=0(z=1)$ but regular at $z=2 y-1$. The above differential equations give

$$
6 g_{7}^{1}-g_{8}^{1}=\left.0\right|_{z=2 y-1} .
$$

In the $y>1$ or $y<0$ regions, the above integrals are regular at $z=1$ and singular at $z=2 y-1$. Thus one can obtain

$$
-2 g_{3}^{1}+6 g_{7}^{1}-g_{8}^{1}=\left.0\right|_{z=1}
$$

Accordingly the boundary condition for $\left\{g_{7}^{1}, g_{8}^{1}\right\}$ is fixed in the entire range of $y$. 
Similar with the above examples, all the remaining unknown boundary conditions can be determined from regular conditions at $z=\{2 y-1,2 y+1, y, 1,0\}$, respectively. We will briefly present the determination of boundary conditions in the following.

As all the integrals are regular at the space-like kinematics point $p_{1}^{2}=-p_{z}^{2}(z=0)$, one can determine the boundary conditions of integrals with the normalization factor $\sqrt{p_{1}^{2}+p_{z}^{2}}$ or $\left(p_{1}^{2}+p_{z}^{2}\right)$. The integrals

$$
\begin{aligned}
& \left\{g_{5}^{1}, g_{7}^{1}, g_{9}^{1}, g_{11}^{1}, g_{13}^{1}, g_{14}^{1}, g_{15}^{1}, g_{16}^{1}, g_{17}^{1}, g_{23}^{1}, g_{24}^{1}, g_{25}^{1}, g_{26}^{1}, g_{28}^{1}, g_{29}^{1}, g_{31}^{1}, g_{32}^{1}, g_{33}^{1}, g_{34}^{1}, g_{35}^{1}, g_{36}^{1}\right\} \\
& \left\{g_{10}^{2}, g_{12}^{2}, g_{14}^{2}, g_{16}^{2}, g_{17}^{2}, g_{19}^{2}, g_{20}^{2}, g_{21}^{2}, g_{23}^{2}, g_{24}^{2}, g_{25}^{2}, g_{26}^{2}, g_{27}^{2}, g_{28}^{2}, g_{29}^{2}, g_{30}^{2}, g_{31}^{2}, g_{32}^{2}\right\} \\
& \left\{g_{7}^{3}, g_{8}^{3}, g_{9}^{3}, g_{10}^{3}, g_{11}^{3}, g_{12}^{3}, g_{15}^{3}, g_{17}^{3}, g_{19}^{3}, g_{21}^{3}, g_{22}^{3}, g_{23}^{3}, g_{25}^{3}, g_{26}^{3}, g_{28}^{3}\right\}
\end{aligned}
$$

vanish at $z=0$. For the integrals without the pre-factor $\sqrt{p_{1}^{2}+p_{z}^{2}}$ or $\left(p_{1}^{2}+p_{z}^{2}\right)$, their boundary conditions should be determined carefully in different regions for $y$.

\subsection{The rest boundary conditions for the integrals in the first family}

In the first family, the $\left\{g_{6}^{1}, g_{8}^{1}, g_{10}^{1}, g_{12}^{1}, g_{21}^{1}, g_{22}^{1}, g_{27}^{1}, g_{30}^{1}\right\}$ do not vanish at $z=0$.

The boundary conditions for $\left\{g_{8}^{1}, g_{30}^{1}\right\}$ are determined from the regularity of the corresponding basis at $z=2 y-1$ in the $(0<y<1)$ region and from the regularity at $z=1$ in the $(y<0, y>1)$ regions. The boundary conditions for $\left\{g_{12}^{1}, g_{27}^{1}\right\}$ can be similarly obtained. But since these integrals have contributions from the same topology with anti-quark contributions, their boundary conditions are determined from the regularity at $z=2 y+1$ in the $(-1<y<0)$ region and from the regularity at $z=1$ for $(y<-1, y>0)$.

To derive the boundary conditions as well as analytic results for the $g_{6}^{1}$ and $g_{10}^{1}$, we consider the region $(0<y<1)$ first. In this region the boundary condition for the $g_{6}^{1}$ is determined from the regularity at $z=2 y-1$, and then one can obtain the corresponding analytic result straightforwardly. In the $y>0$ region, $g_{10}^{1}$ is both singular at $z=2 y+1$ and $z=1$, and the boundary condition can be determined from the differential equations of $g_{15}^{1}$ at $z=y$ :

$$
\frac{\partial g_{15}^{1}}{\partial z}=\epsilon \frac{-4 g_{5}^{1}+g_{6}^{1}-4 g_{9}^{1}+g_{10}^{1}-4 g_{15}^{1}-4 g_{16}^{1}}{4(z-y)}+\ldots
$$

where the ellipses stand for less singular terms at $z=y$. Since the $p_{1}^{2}=\left(y^{2}-1\right) p_{z}^{2}(z=y)$ is space-like, and $g_{15}^{1}$ is regular at $p_{1}^{2}=\left(y^{2}-1\right) p_{z}^{2}$, one has

$$
g_{10}^{1}=-\left.\left(-4 g_{5}^{1}+g_{6}^{1}-4 g_{9}^{1}-4 g_{15}^{1}-4 g_{16}^{1}\right)\right|_{z=y} .
$$

Then we can obtain the analytic results for the $g_{10}^{1}$ in the $0<y<1$ region and the results can be extrapolated to the $y>0$ region. In the $y<-1$ region, the results of $g_{10}^{1}$ can be obtained with the replacement $(y \rightarrow-y-1)$ from the ones in the $y>0$ region, with an overall minus sign. For the $(-1<y<0)$, the $g_{10}^{1}$ is singular at $z=1$ but regular at $z=2 y+1$, and thus we can determine the boundary condition at $z=2 y+1$. This completes the boundary conditions for the $g_{10}^{1}$ in the entire range of $y$. Results for the $g_{6}^{1}$ in the $(y<0, y>1)$ regions can be derived from the ones for $g_{10}^{1}$ in the region $(y<-1, y>0)$ through the replacement $y \rightarrow y-1$. 
The base $g_{22}^{1}$ depends on $y$ only, in the region $(y<-1, y>0)$ it can be determined from the regularity of $g_{26}^{1}$ at $z=1$, whose differential equation is given as

$$
\frac{\partial g_{26}^{1}}{\partial z}=\epsilon \frac{2 g_{1}^{1}-2 g_{11}^{1}+g_{12}^{1}-2 g_{20}^{1}-g_{22}^{1}-2 g_{24}^{1}-8 g_{26}^{1}}{4(z-1)}+\ldots
$$

and the results of $g_{22}^{1}$ in $y>0$ region can be extrapolated to the $y>-1$ region. The results for the $g_{21}^{1}$ are related to the ones for the $g_{22}^{1}$ through $y \rightarrow y-1$.

\subsection{The rest boundary conditions for the integrals in the second family}

For the second family, the boundary conditions for $\left\{g_{9}^{2}, g_{11}^{2}, g_{13}^{2}, g_{15}^{2}, g_{18}^{2}, g_{22}^{2}\right\}$ are to be determined. The boundary conditions for the $g_{11}^{2}$ and $g_{15}^{2}$ as well as full analytic results can be obtained from the ones in the first family through the symmetry relations in eq. (3.11). Results for the $g_{9}^{2}$ can be derived from $g_{22}^{1}$ by reversing the sign for the variable $y$.

The boundary for the $\left\{g_{13}^{2}, g_{18}^{2}, g_{22}^{2}\right\}$ can be determined from the regularity at $z=2 y-1$ for $(0<y<1)$ region and from the regularity at $z=1$ for $(y<0, y>1)$.

\subsection{The rest boundary conditions for the integrals in the third family}

For the third family, the boundary conditions for $\left\{g_{16}^{3}, g_{18}^{3}, g_{20}^{3}, g_{24}^{3}, g_{27}^{3}\right\}$ need to be determined. Their boundary conditions as well as full analytic results can be obtained from the first and second families using the symmetry relations in eq. (3.11).

The analytic results for all the canonical basis in the entire range of $y$ can be found in the Supplementary material of this work. The explicit expressions of boundary conditions can be obtained directly from the analytic results.

\section{Analytic results and validations}

In the Lattice QCD study of quasi PDFs, the RI/MOM scheme is often adopted to renormalize the bare quantities [80]. In this scheme, a counterterm with off-shell quarks is introduced and thus in this work, we will present the analytic results with both off-shell and on-shell quarks.

\subsection{Results for off-shell quarks}

After determining all the boundary conditions, we can obtain the analytic results for all integrals in the entire range of $y$. We calculate all the integrals up to weight-3, which are required for the involved two-loop corrections. For the first family, we can divide the $y$ range into 4 regions $(y<-1,-1<y<0,0<y<1, y>1)$, while for the second and third family, the results are divided into 3 regions $(y<0,0<y<1, y>1)$. 
For the convenience of the reader, we show the analytic results of some typical integrals at $(0<y<1)$ as follows:

$$
\begin{aligned}
& g_{5}^{1}=\epsilon\left[G_{1}(z)-G_{-1}(z)\right] \\
& +\epsilon^{2}\left[2 G_{1-2 y,-1}(z)-2 G_{1-2 y, 1}(z)+2 G_{2 y-1,-1}(z)-2 G_{2 y-1,1}(z)\right. \\
& +G_{-1,-1}(z)-G_{-1,1}(z)-2 G_{0,-1}(z)+2 G_{0,1}(z)+G_{1,-1}(z)-G_{1,1}(z) \\
& +\left(G_{-1}(z)-G_{1}(z)\right)\left(\ln \left(16 y^{2}\right)+\ln \left((y-1)^{2}\right)\right) \\
& \left.-\left(G_{1-2 y}(z)-G_{2 y-1}(z)\right)\left(\ln \left((y-1)^{2}\right)-\ln \left(y^{2}\right)\right)\right]+\mathcal{O}\left(\epsilon^{3}\right), \\
& g_{7}^{1}=\epsilon\left[G_{1}(z)-G_{-1}(z)\right] \\
& +\epsilon^{2}\left[2 G_{-1,-1}(z)-2 G_{1,1}(z)-G_{-1,1}(z)+G_{1,-1}(z)-2 G_{0,-1}(z)+2 G_{0,1}(z)\right. \\
& +2 G_{1-2 y,-1}(z)-G_{1-2 y, 1}(z)+G_{2 y-1,-1}(z)-2 G_{2 y-1,1}(z) \\
& +\left(G_{1}(z)-G_{-1}(z)+G_{2 y-1}(z)-G_{1-2 y}(z)\right)\left(\ln (2)+\ln \left((y-1)^{2}\right)\right) \\
& -\left(G_{-1}(z)-G_{1}(z)\right)\left(4 \ln (2)+2 \ln \left((y-1)^{2}\right)-\frac{1}{2} \ln \left(y^{2}\right)\right) \\
& \left.+\frac{1}{2}\left(G_{1-2 y}(z)-G_{2 y-1}(z)\right) \ln \left(y^{2}\right)\right]+\mathcal{O}\left(\epsilon^{3}\right), \\
& g_{35}^{1}=\epsilon^{3}\left[(\ln (1-y)-\ln (y)+\ln (2 y+1))\left(2 G_{1,-1}(z)+2 G_{-1,1}(z)-2 G_{-1,-1}(z)-2 G_{1,1}(z)\right)\right. \\
& +(\ln (1-y)+\ln (y)+2 \ln (2 y+1))\left(G_{1,2 y-1}(z)+G_{-1,1-2 y}(z)-G_{-1,2 y-1}(z)-G_{1,1-2 y}(z)\right) \\
& +2 \ln (2 y+1)\left(G_{1-2 y,-y}(z)+G_{2 y-1, y}(z)-G_{1, y}(z)-G_{-1,-y}(z)\right) \\
& +(\ln (1-y)+\ln (y))\left(G_{2 y-1,1}(z)-G_{2 y-1,-1}(z)+G_{1-2 y,-1}(z)-G_{1-2 y, 1}(z)\right) \\
& +3 G_{-1,-1,-1}(z)-G_{-1,-1,1}(z)-2 G_{-1,-1,-2 y-1}(z) \\
& -G_{-1,1,-1}(z)-G_{-1,1,1}(z)+2 G_{-1,1,-2 y-1}(z)-G_{-1,1-2 y,-1}(z)-G_{-1,1-2 y, 1}(z) \\
& +2 G_{-1,1-2 y,-2 y-1}(z)+2 G_{-1,-y, 1}(z)-2 G_{-1,-y,-2 y-1}(z)+G_{-1,2 y-1,-1}(z) \\
& +G_{-1,2 y-1,1}(z)-2 G_{-1,2 y-1,2 y+1}(z)-G_{1,-1,-1}(z)-G_{1,-1,1}(z)+2 G_{1,-1,2 y+1}(z) \\
& -G_{1,1,-1}(z)+3 G_{1,1,1}(z)-2 G_{1,1,2 y+1}(z)+G_{1,1-2 y,-1}(z)+G_{1,1-2 y, 1}(z) \\
& -2 G_{1,1-2 y,-2 y-1}(z)+2 G_{1, y,-1}(z)-2 G_{1, y, 2 y+1}(z)-G_{1,2 y-1,-1}(z) \\
& -G_{1,2 y-1,1}(z)+2 G_{1,2 y-1,2 y+1}(z)-2 G_{1-2 y,-1,-1}(z)+2 G_{1-2 y,-1,1}(z) \\
& -2 G_{1-2 y, 1,-2 y-1}(z)+2 G_{1-2 y, 1,2 y+1}(z)-2 G_{1-2 y,-y, 1}(z)+2 G_{1-2 y,-y,-2 y-1}(z) \\
& +2 G_{2 y-1,-1,-2 y-1}(z)-2 G_{2 y-1,-1,2 y+1}(z)+2 G_{2 y-1,1,-1}(z)-2 G_{2 y-1,1,1}(z) \\
& \left.-2 G_{2 y-1, y,-1}(z)+2 G_{2 y-1, y, 2 y+1}(z)\right]+\mathcal{O}\left(\epsilon^{4}\right) .
\end{aligned}
$$

The Goncharov polylogarithms (GPLs) [81] in the above expressions are defined as follows

$$
\begin{aligned}
G_{a_{1}, a_{2}, \ldots, a_{n}}(y) & \equiv \int_{0}^{y} \frac{\mathrm{d} t}{t-a_{1}} G_{a_{2}, \ldots, a_{n}}(t), \\
G_{\overrightarrow{0}_{n}}(y) & \equiv \frac{1}{n !} \ln ^{n} y .
\end{aligned}
$$


These functions can be viewed as a special case belonging to a more general type of integrals called Chen-iterated integrals [82]. When all the index $a_{i}$ belong to the set $\{0, \pm 1\}$, the Goncharov polylogarithms can be transformed into the well-known Harmonic polylogarithms (HPLs) [83] as

$$
\begin{aligned}
H_{\overrightarrow{0}_{n}}(y) & =G_{\overrightarrow{0}_{n}}(y), \\
H_{a_{1}, a_{2}, \ldots, a_{n}}(y) & =(-1)^{k} G_{a_{1}, a_{2}, \ldots, a_{n}}(y),
\end{aligned}
$$

where $k$ equals to the times of element $(+1)$ taken in $\left(a_{1}, a_{2}, \ldots, a_{n}\right)$.

The GPLs fulfil the following shuffle rules

$$
G_{a_{1}, \ldots, a_{m}}(y) G_{b_{1}, \ldots, b_{n}}(y)=\sum_{c \in a \amalg b} G_{c_{1}, c_{2}, \ldots, c_{m+n}}(y) .
$$

Here, $a \amalg b$ is composed of the shuffle products of list $a$ and $b$. It is defined as the set of the lists containing all the elements of $a$ and $b$, with the ordering of the elements of $a$ and $b$ preserved. The GPLs and HPLs can be numerically evaluated within the GINAC implementation [84, 85]. Mathematica package HPL [86, 87] is available to reduce and evaluate the HPLs. Up to weight four, the GPLs and HPLs can be transformed to the functions of $\ln , \mathrm{Li}_{n}$ and $\mathrm{Li}_{22}$, with the algorithms and packages described in [88].

\subsection{Results for on-shell quarks}

To perform the matching between quasi and lightcone PDFs in the $\overline{\mathrm{MS}}$ scheme, one also needs the integrals results at $p_{1}^{2}=0(z=1)$. In this subsection, we present the calculation of integrals for $p_{1}^{2}=0(z=1)$. For $p_{1}^{2}=0(z=1)$, there are 14 independent integrals for the first family. There are 13 integrals for the second family, and 12 integrals for the third family. As some integrals such as $g_{7}^{1}$ are singular at $p_{1}^{2}=0(z=1)$, we use the method in ref. [74] to extract their analytic results from $p_{1}^{2} \neq 0(z \neq 1)$. For $p_{1}^{2}=0(z=1)$, we will need to calculate the following basis

$$
\begin{aligned}
& \left\{g_{1}^{1}, g_{2}^{1}, g_{3}^{1}, g_{7}^{1}, g_{11}^{1}, g_{18}^{1}, g_{19}^{1}, g_{20}^{1}, g_{21}^{1}, g_{22}^{1}, g_{24}^{1}, g_{25}^{1}, g_{28}^{1}, g_{29}^{1}\right\} \\
& \left\{g_{1}^{2}, g_{2}^{2}, g_{3}^{2}, g_{4}^{2}, g_{7}^{2}, g_{8}^{2}, g_{9}^{2}, g_{10}^{2}, g_{12}^{2}, g_{16}^{2}, g_{17}^{2}, g_{19}^{2}, g_{21}^{2}\right\} \\
& \left\{g_{1}^{3}, g_{2}^{3}, g_{4}^{3}, g_{5}^{3}, g_{13}^{3}, g_{14}^{3}, g_{15}^{3}, g_{19}^{3}, g_{22}^{3}, g_{23}^{3}, g_{25}^{3}, g_{26}^{3}\right\}
\end{aligned}
$$

For illustration, we show the analytic results for $g_{7}^{1}$ in the $(0<y<1)$ region as

$$
\begin{aligned}
\left.g_{7}^{1}\right|_{0<y<1}= & \frac{1}{2}+\epsilon\left(-\frac{1}{2} \ln (y)-\ln (1-y)-2 \ln (2)\right) \\
& +\epsilon^{2}\left(\frac{1}{2}\left(2 \operatorname{Li}_{2}(y)+4 \ln ^{2}(1-y)+8 \ln (2) \ln (1-y)+\frac{3 \ln ^{2}(y)}{2}+4 \ln (2) \ln (y)\right)\right. \\
& \left.+\frac{5 \pi^{2}}{12}+4 \ln ^{2}(2)\right)+\epsilon^{3} \frac{1}{12}\left(-48 \operatorname{Li}_{3}(1-y)-36 \operatorname{Li}_{3}(y)-48 \operatorname{Li}_{2}(y) \ln (2-2 y)\right. \\
& -96 \ln ^{2}(2) \ln (1-y)-48 \ln ^{2}(2) \ln (y)-96 \ln (2) \ln ^{2}(1-y)-36 \ln (2) \ln ^{2}(y) \\
& -32 \ln ^{3}(1-y)-9 \ln ^{3}(y)-2 \pi^{2} \ln (1-y)-24 \ln ^{2}(1-y) \ln (y)-5 \pi^{2} \ln (y) \\
& \left.-52 \zeta(3)-64 \ln ^{3}(2)-20 \pi^{2} \ln (2)\right)+\mathcal{O}\left(\epsilon^{4}\right),
\end{aligned}
$$


while the analytic results for $g_{7}^{1}$ in the $(y>1)$ region are

$$
\begin{aligned}
\left.g_{7}^{1}\right|_{y>1}= & \epsilon(\ln (y-1)-\ln (y)) \\
& +\epsilon^{2}\left(\operatorname{Li}_{2}\left(\frac{1}{y}\right)+2 \ln ^{2}(y)-2 \ln ^{2}(y-1)+4 \ln (2)(\ln (y)-\ln (y-1))\right) \\
& +\epsilon^{3}\left(4 \operatorname{Li}_{3}\left(\frac{1}{1-y}\right)+3 \operatorname{Li}_{3}\left(\frac{1}{y}\right)-4 \operatorname{Li}_{2}\left(\frac{1}{y}\right) \ln (2 y-2)-2 \ln ^{3}(y)+2 \ln ^{3}(y-1)\right. \\
& +2 \ln ^{2}(y-1) \ln (y)-2 \ln ^{2}(y) \ln (y-1)-8 \ln (2) \ln ^{2}(y)+8 \ln (2) \ln ^{2}(y-1) \\
& \left.+\left(\frac{5}{6} \pi^{2}+8 \ln ^{2}(2)\right)(\ln (y-1)-\ln (y))\right)+\mathcal{O}\left(\epsilon^{4}\right) .
\end{aligned}
$$

The analytic results for $g_{7}^{1}$ in the $(y<0)$ region are

$$
\begin{aligned}
\left.g_{7}^{1}\right|_{y<0}= & \epsilon(\ln (-y)-\ln (1-y)) \\
& +\epsilon^{2}\left(-\operatorname{Li}_{2}\left(\frac{1}{y}\right)-2 \ln ^{2}(-y)+2 \ln ^{2}(1-y)+4 \ln (2)(\ln (1-y)-\ln (-y))\right) \\
& -\epsilon^{3}\left(4 \operatorname{Li}_{3}\left(\frac{1}{1-y}\right)+3 \operatorname{Li}_{3}\left(\frac{1}{y}\right)-4 \operatorname{Li}_{2}\left(\frac{1}{y}\right) \ln (2-2 y)-2 \ln ^{3}(-y)+2 \ln ^{3}(1-y)\right. \\
& +2 \ln ^{2}(1-y) \ln (-y)-2 \ln ^{2}(-y) \ln (1-y)-8 \ln (2) \ln ^{2}(-y)+8 \ln (2) \ln ^{2}(1-y) \\
& \left.+\left(\frac{5}{6} \pi^{2}+8 \ln ^{2}(2)\right)(\ln (1-y)-\ln (-y))\right)+\mathcal{O}\left(\epsilon^{4}\right) .
\end{aligned}
$$

\subsection{Validations}

All the analytic results have been checked with the numerical packages FIESTA [89, 90], and we find perfect agreements between the numerical results and our analytic calculations. For illustration, we show the test of integrals $g_{7}^{1}=\epsilon^{2} \sqrt{p_{1}^{2}+p_{z}^{2}} I_{1,1,0,0,2,1,0}^{1}$, and the results up to $\mathcal{O}(\epsilon)$ at $\left(p_{1}^{2}=-\frac{1}{2}, y=\frac{1}{3}, p_{z}=1\right)$ are given as

Analytic:

$$
I_{1,1,0,0,2,1,0}^{1}=\frac{-2.492900960}{\epsilon}+0.4498613241+\epsilon(-21.287203876),
$$

FIESTA:

$$
I_{1,1,0,0,2,1,0}^{1}=\frac{-2.49290 \pm 0.0000652}{\epsilon}+0.449836 \pm 0.000847+\epsilon(-21.2872 \pm 0.004169) .
$$

For the on-shell quark case, the results for $I_{1,1,0,0,2,1,0}^{1}$ at $\left(p_{1}^{2}=0, y=\frac{1}{3}, p_{z}=1\right)$ have $\frac{1}{\epsilon^{2}}$ pole and are expressed as

Analytic:

$$
I_{1,1,0,0,2,1,0}^{1}=\frac{0.5}{\epsilon^{2}}+\frac{-0.4315231087}{\epsilon}+4.9871880743+\epsilon(-15.840344856),
$$

FIESTA:

$$
\begin{aligned}
I_{1,1,0,0,2,1,0}^{1}= & \frac{0.5 \pm 0.000017}{\epsilon^{2}}+\frac{-0.431509 \pm 0.000130}{\epsilon}+4.98750 \pm 0.001077 \\
& +\epsilon(-15.8377 \pm 0.007512)
\end{aligned}
$$


The results at $\left(p_{1}^{2}=0, y=1.35, p_{z}=1\right)$ are

Analytic:

$I_{1,1,0,0,2,1,0}^{1}=\frac{-1.349926717}{\epsilon}+2.680139987+\epsilon(-17.35111093)$,

FIESTA:

$I_{1,1,0,0,2,1,0}^{1}=\frac{-1.34992 \pm 0.00004}{\epsilon}+2.68014 \pm 0.00023+\epsilon(-17.3511 \pm 0.0007)$,

while the results at $\left(p_{1}^{2}=0, y=-0.35, p_{z}=1\right)$ are

Analytic:

$I_{1,1,0,0,2,1,0}^{1}=\frac{-1.349926717}{\epsilon}+3.591291058+\epsilon(-16.19882013)$,

FIESTA:

$I_{1,1,0,0,2,1,0}^{1}=\frac{-1.34993 \pm 0.00003}{\epsilon}+3.59129 \pm 0.00015+\epsilon(-16.1988 \pm 0.0007)$.

One can see from the above comparison that the numerical results obtained with the FIESTA perfectly agree with the analytic results.

\section{Discussions and conclusions}

In summary, we have presented the detailed results for the two-loop master integrals of NNLO corrections to quark quasi PDFs. Three families of master integrals are derived. Making use of the method of differential equations along with the choice of canonical basis, we obtain the analytic results for all the master integrals in terms of Goncharov polylogarithms and polylogarithms. Our analytic results are in agreement with the numerical results by FIESTA $[89,90]$ package in the entire range of $y$. These two-loop master integrals are helpful to extract the two-loop matching coefficients between quasi and lightcone PDFs, and accordingly together with the Lattice QCD simulations deepen understanding the lightcone structures inside a hadron. These results have been sued to calculate the NNLO corrections to quasi PDFs distributions for valence quarks [91].

Based on the previous calculation of perturbative kernel at one-loop accuracy, we find that the PDFs for quarks, gluons, meson distribution amplitudes and generalized parton distributions have used some common integrals. Thus from this viewpoint our results in this work with some generalizations are also likely useful in the studies of these quantities.

\section{Acknowledgments}

We thank Feng Yuan and Xiangdong Ji for valuable discussions. LBC is supported by the National Natural Science Foundation of China (NSFC) under the grant No. 11805042. WW is supported by NSFC under grants No. 11735010, 11911530088, U2032102, by Natural Science Foundation of Shanghai under grant No. 15DZ2272100. RLZ is supported by NSFC under grant No. 11705092, 12075124, by Natural Science Foundation of Jiangsu 
under Grant No. BK20171471 and Jiangsu Qing Lan Project, by China Scholarship Council under Grant No. 201906865014 and partially supported by the U.S. Department of Energy, Office of Science, Office of Nuclear Physics, under contract number DE-AC02-05CH11231.

Open Access. This article is distributed under the terms of the Creative Commons Attribution License (CC-BY 4.0), which permits any use, distribution and reproduction in any medium, provided the original author(s) and source are credited.

\section{References}

[1] LHPC and TXL collaborations, Moments of nucleon light cone quark distributions calculated in full lattice QCD, Phys. Rev. D 66 (2002) 034506 [hep-lat/0201021] [INSPIRE].

[2] X. Ji, Parton Physics on a Euclidean Lattice, Phys. Rev. Lett. 110 (2013) 262002 [arXiv: 1305.1539] [INSPIRE].

[3] X. Ji, Parton Physics from Large-Momentum Effective Field Theory, Sci. China Phys. Mech. Astron. 57 (2014) 1407 [arXiv: 1404.6680] [INSPIRE].

[4] Y.-Q. Ma and J.-W. Qiu, Extracting Parton Distribution Functions from Lattice QCD Calculations, Phys. Rev. D 98 (2018) 074021 [arXiv:1404.6860] [INSPIRE].

[5] Y.-Q. Ma and J.-W. Qiu, Exploring Partonic Structure of Hadrons Using ab initio Lattice QCD Calculations, Phys. Rev. Lett. 120 (2018) 022003 [arXiv:1709.03018] [INSPIRE].

[6] A.V. Radyushkin, Quasi-parton distribution functions, momentum distributions, and pseudo-parton distribution functions, Phys. Rev. D 96 (2017) 034025 [arXiv:1705.01488] [INSPIRE].

[7] X. Xiong, X. Ji, J.-H. Zhang and Y. Zhao, One-loop matching for parton distributions: Nonsinglet case, Phys. Rev. D 90 (2014) 014051 [arXiv:1310.7471] [INSPIRE].

[8] X. Ji and J.-H. Zhang, Renormalization of quasiparton distribution, Phys. Rev. D 92 (2015) 034006 [arXiv: 1505.07699] [INSPIRE].

[9] X. Ji, A. Schäfer, X. Xiong and J.-H. Zhang, One-Loop Matching for Generalized Parton Distributions, Phys. Rev. D 92 (2015) 014039 [arXiv:1506.00248] [INSPIRE].

[10] X. Xiong and J.-H. Zhang, One-loop matching for transversity generalized parton distribution, Phys. Rev. D 92 (2015) 054037 [arXiv: 1509.08016] [INSPIRE].

[11] X. Ji, P. Sun, X. Xiong and F. Yuan, Soft factor subtraction and transverse momentum dependent parton distributions on the lattice, Phys. Rev. D 91 (2015) 074009 [arXiv: 1405.7640] [INSPIRE].

[12] C. Monahan, Smeared quasidistributions in perturbation theory, Phys. Rev. D 97 (2018) 054507 [arXiv: 1710.04607] [INSPIRE].

[13] X. Ji, L.-C. Jin, F. Yuan, J.-H. Zhang and Y. Zhao, Transverse momentum dependent parton quasidistributions, Phys. Rev. D 99 (2019) 114006 [arXiv:1801.05930] [InSPIRE].

[14] I.W. Stewart and Y. Zhao, Matching the quasiparton distribution in a momentum subtraction scheme, Phys. Rev. D 97 (2018) 054512 [arXiv: 1709.04933] [INSPIRE].

[15] M. Constantinou and H. Panagopoulos, Perturbative renormalization of quasi-parton distribution functions, Phys. Rev. D 96 (2017) 054506 [arXiv: 1705.11193] [InSPIRE]. 
[16] J. Green, K. Jansen and F. Steffens, Nonperturbative Renormalization of Nonlocal Quark Bilinears for Parton Quasidistribution Functions on the Lattice Using an Auxiliary Field, Phys. Rev. Lett. 121 (2018) 022004 [arXiv:1707.07152] [INSPIRE].

[17] T. Izubuchi, X. Ji, L. Jin, I.W. Stewart and Y. Zhao, Factorization Theorem Relating Euclidean and Light-Cone Parton Distributions, Phys. Rev. D 98 (2018) 056004 [arXiv: 1801.03917] [INSPIRE].

[18] X. Xiong, T. Luu and U.-G. Meißner, Quasi-Parton Distribution Function in Lattice Perturbation Theory, arXiv:1705.00246 [INSPIRE].

[19] W. Wang, S. Zhao and R. Zhu, Gluon quasidistribution function at one loop, Eur. Phys. J. C 78 (2018) 147 [arXiv:1708.02458] [INSPIRE].

$[20] \mathrm{W}$. Wang and S. Zhao, On the power divergence in quasi gluon distribution function, JHEP 05 (2018) 142 [arXiv:1712.09247] [INSPIRE].

[21] J. Xu, Q.-A. Zhang and S. Zhao, Light-cone distribution amplitudes of vector meson in a large momentum effective theory, Phys. Rev. D 97 (2018) 114026 [arXiv:1804.01042] [INSPIRE].

[22] J.-W. Chen, S.D. Cohen, X. Ji, H.-W. Lin and J.-H. Zhang, Nucleon Helicity and Transversity Parton Distributions from Lattice QCD, Nucl. Phys. B 911 (2016) 246 [arXiv: 1603.06664] [INSPIRE].

[23] J.-H. Zhang, J.-W. Chen, X. Ji, L. Jin and H.-W. Lin, Pion Distribution Amplitude from Lattice QCD, Phys. Rev. D 95 (2017) 094514 [arXiv:1702.00008] [InSPIRE].

[24] T. Ishikawa, Y.-Q. Ma, J.-W. Qiu and S. Yoshida, Practical quasi parton distribution functions, arXiv:1609.02018 [INSPIRE].

[25] J.-W. Chen, X. Ji and J.-H. Zhang, Improved quasi parton distribution through Wilson line renormalization, Nucl. Phys. B 915 (2017) 1 [arXiv: 1609.08102] [INSPIRE].

[26] X. Ji, J.-H. Zhang and Y. Zhao, Renormalization in Large Momentum Effective Theory of Parton Physics, Phys. Rev. Lett. 120 (2018) 112001 [arXiv:1706.08962] [INSPIRE].

[27] T. Ishikawa, Y.-Q. Ma, J.-W. Qiu and S. Yoshida, Renormalizability of quasiparton distribution functions, Phys. Rev. D 96 (2017) 094019 [arXiv:1707.03107] [InSPIRE].

[28] J.-W. Chen et al., Parton distribution function with nonperturbative renormalization from lattice QCD, Phys. Rev. D 97 (2018) 014505 [arXiv:1706.01295] [InSPIRE].

[29] C. Alexandrou et al., A complete non-perturbative renormalization prescription for quasi-PDFs, Nucl. Phys. B 923 (2017) 394 [arXiv:1706.00265] [INSPIRE].

[30] LP3 collaboration, Symmetry properties of nonlocal quark bilinear operators on a Lattice, Chin. Phys. C 43 (2019) 103101 [arXiv:1710.01089] [InSPIRE].

[31] LP3 collaboration, Improved parton distribution functions at the physical pion mass, Phys. Rev. D 98 (2018) 054504 [arXiv: 1708.05301] [INSPIRE].

[32] T. Ishikawa et al., Gaussian-weighted parton quasi-distribution (Lattice Parton Physics Project (LP $)$ ), Sci. China Phys. Mech. Astron. 62 (2019) 991021 [arXiv:1711.07858] [INSPIRE].

[33] H.-n. Li, Nondipolar Wilson links for quasiparton distribution functions, Phys. Rev. D 94 (2016) 074036 [arXiv:1602.07575] [INSPIRE]. 
[34] C. Monahan and K. Orginos, Quasi parton distributions and the gradient flow, JHEP 03 (2017) 116 [arXiv:1612.01584] [INSPIRE].

[35] A. Radyushkin, Nonperturbative Evolution of Parton Quasi-Distributions, Phys. Lett. B 767 (2017) 314 [arXiv:1612.05170] [INSPIRE].

[36] G.C. Rossi and M. Testa, Note on lattice regularization and equal-time correlators for parton distribution functions, Phys. Rev. D 96 (2017) 014507 [arXiv:1706.04428] [InSPIRE].

[37] C.E. Carlson and M. Freid, Lattice corrections to the quark quasidistribution at one-loop, Phys. Rev. D 95 (2017) 094504 [arXiv: 1702.05775] [InSPIRE].

[38] X. Ji, J.-H. Zhang and Y. Zhao, More On Large-Momentum Effective Theory Approach to Parton Physics, Nucl. Phys. B 924 (2017) 366 [arXiv:1706.07416] [InSPIRE].

[39] R.A. Briceño, J.V. Guerrero, M.T. Hansen and C.J. Monahan, Finite-volume effects due to spatially nonlocal operators, Phys. Rev. D 98 (2018) 014511 [arXiv:1805.01034] [InSPIRE].

[40] T.J. Hobbs, Quantifying finite-momentum effects in the quark quasidistribution functions of mesons, Phys. Rev. D 97 (2018) 054028 [arXiv: 1708.05463] [InSPIRE].

[41] Y. Jia, S. Liang, L. Li and X. Xiong, Solving the Bars-Green equation for moving mesons in two-dimensional QCD, JHEP 11 (2017) 151 [arXiv:1708.09379] [INSPIRE].

[42] S.-S. Xu, L. Chang, C.D. Roberts and H.-S. Zong, Pion and kaon valence-quark parton quasidistributions, Phys. Rev. D 97 (2018) 094014 [arXiv:1802.09552] [INSPIRE].

[43] Y. Jia, S. Liang, X. Xiong and R. Yu, Partonic quasidistributions in two-dimensional QCD, Phys. Rev. D 98 (2018) 054011 [arXiv: 1804.04644] [InSPIRE].

[44] G. Spanoudes and H. Panagopoulos, Renormalization of Wilson-line operators in the presence of nonzero quark masses, Phys. Rev. D 98 (2018) 014509 [arXiv:1805.01164] [INSPIRE].

[45] G. Rossi and M. Testa, Euclidean versus Minkowski short distance, Phys. Rev. D 98 (2018) 054028 [arXiv: 1806.00808] [INSPIRE].

[46] LATTICE PARTON collaboration, Unpolarized isovector quark distribution function from lattice QCD: A systematic analysis of renormalization and matching, Phys. Rev. D 101 (2020) 034020 [arXiv: 1807.06566] [INSPIRE].

[47] X. Ji, Y. Liu and I. Zahed, Quasiparton distribution functions: Two-dimensional scalar and spinor QCD, Phys. Rev. D 99 (2019) 054008 [arXiv: 1807.07528] [INSPIRE].

[48] S. Bhattacharya, C. Cocuzza and A. Metz, Generalized quasi parton distributions in a diquark spectator model, Phys. Lett. B 788 (2019) 453 [arXiv:1808.01437] [InSPIRE].

[49] A.V. Radyushkin, Structure of parton quasi-distributions and their moments, Phys. Lett. B 788 (2019) 380 [arXiv: 1807.07509] [INSPIRE].

[50] J.-H. Zhang, X. Ji, A. Schäfer, W. Wang and S. Zhao, Accessing Gluon Parton Distributions in Large Momentum Effective Theory, Phys. Rev. Lett. 122 (2019) 142001 [arXiv: 1808.10824] [INSPIRE].

[51] Z.-Y. Li, Y.-Q. Ma and J.-W. Qiu, Multiplicative Renormalizability of Operators defining Quasiparton Distributions, Phys. Rev. Lett. 122 (2019) 062002 [arXiv:1809.01836] [INSPIRE]. 
[52] V.M. Braun, A. Vladimirov and J.-H. Zhang, Power corrections and renormalons in parton quasidistributions, Phys. Rev. D 99 (2019) 014013 [arXiv: 1810.00048] [InSPIRE].

[53] Y.-S. Liu, W. Wang, J. Xu, Q.-A. Zhang, S. Zhao and Y. Zhao, Matching the meson quasidistribution amplitude in the RI/MOM scheme, Phys. Rev. D 99 (2019) 094036 [arXiv: 1810.10879] [INSPIRE].

[54] M.A. Ebert, I.W. Stewart and Y. Zhao, Determining the Nonperturbative Collins-Soper Kernel From Lattice QCD, Phys. Rev. D 99 (2019) 034505 [arXiv:1811.00026] [InSPIRE].

[55] M.A. Ebert, I.W. Stewart and Y. Zhao, Towards Quasi-Transverse Momentum Dependent PDFs Computable on the Lattice, JHEP 09 (2019) 037 [arXiv:1901.03685] [INSPIRE].

[56] M. Constantinou, H. Panagopoulos and G. Spanoudes, One-loop renormalization of staple-shaped operators in continuum and lattice regularizations, Phys. Rev. D 99 (2019) 074508 [arXiv: 1901.03862] [INSPIRE].

[57] Y.-S. Liu et al., Matching generalized parton quasidistributions in the RI/MOM scheme, Phys. Rev. D 100 (2019) 034006 [arXiv: 1902.00307] [INSPIRE].

[58] S. Bhattacharya, C. Cocuzza and A. Metz, Exploring twist-2 GPDs through quasi-distributions in a diquark spectator model, Phys. Rev. D 102 (2020) 054021 [arXiv: 1903.05721] [INSPIRE].

[59] W. Wang, J.-H. Zhang, S. Zhao and R. Zhu, Complete matching for quasidistribution functions in large momentum effective theory, Phys. Rev. D 100 (2019) 074509 [arXiv: 1904.00978] [INSPIRE].

[60] V.M. Braun, K.G. Chetyrkin and B.A. Kniehl, Renormalization of parton quasi-distributions beyond the leading order: spacelike vs. timelike, JHEP 07 (2020) 161 [arXiv:2004.01043] [INSPIRE].

[61] S. Bhattacharya, K. Cichy, M. Constantinou, A. Metz, A. Scapellato and F. Steffens, New insights on proton structure from lattice QCD: the twist-3 parton distribution function $g_{T}(x)$, arXiv: 2004.04130 [INSPIRE].

[62] S. Bhattacharya, K. Cichy, M. Constantinou, A. Metz, A. Scapellato and F. Steffens, One-loop matching for the twist-3 parton distribution $g_{T}(x)$, Phys. Rev. D 102 (2020) 034005 [arXiv: 2005.10939] [INSPIRE].

[63] L.-B. Chen, W. Wang and R. Zhu, Quasi parton distribution functions at NNLO: flavor non-diagonal quark contributions, Phys. Rev. D 102 (2020) 011503 [arXiv:2005.13757] [INSPIRE].

[64] R. Zhang, C. Honkala, H.-W. Lin and J.-W. Chen, Pion and Kaon Distribution Amplitudes in the Continuum Limit, arXiv:2005.13955 [INSPIRE].

[65] S. Dulat et al., New parton distribution functions from a global analysis of quantum chromodynamics, Phys. Rev. D 93 (2016) 033006 [arXiv: 1506.07443] [InSPIRE].

[66] K. Cichy and M. Constantinou, A guide to light-cone PDFs from Lattice QCD: an overview of approaches, techniques and results, Adv. High Energy Phys. 2019 (2019) 3036904 [arXiv: 1811.07248] [INSPIRE].

[67] X. Ji, Y.-S. Liu, Y. Liu, J.-H. Zhang and Y. Zhao, Large-Momentum Effective Theory, arXiv: 2004.03543 [INSPIRE]. 
[68] A.V. Kotikov, Differential equations method: New technique for massive Feynman diagrams calculation, Phys. Lett. B 254 (1991) 158 [INSPIRE].

[69] A.V. Kotikov, Differential equation method: The calculation of $N$ point Feynman diagrams, Phys. Lett. B 267 (1991) 123 [Erratum ibid. 295 (1992) 409] [INSPIRE].

[70] E. Remiddi, Differential equations for Feynman graph amplitudes, Nuovo Cim. A 110 (1997) 1435 [hep-th/9711188] [INSPIRE].

[71] T. Gehrmann and E. Remiddi, Differential equations for two loop four point functions, Nucl. Phys. B $\mathbf{5 8 0}$ (2000) 485 [hep-ph/9912329] [InSPIRE].

[72] M. Argeri and P. Mastrolia, Feynman Diagrams and Differential Equations, Int. J. Mod. Phys. A 22 (2007) 4375 [arXiv: 0707.4037] [INSPIRE].

[73] J.M. Henn, Multiloop integrals in dimensional regularization made simple, Phys. Rev. Lett. 110 (2013) 251601 [arXiv: 1304.1806] [INSPIRE].

[74] J.M. Henn, A.V. Smirnov and V.A. Smirnov, Evaluating single-scale and/or non-planar diagrams by differential equations, JHEP 03 (2014) 088 [arXiv: 1312.2588] [INSPIRE].

[75] J.M. Henn, Lectures on differential equations for Feynman integrals, J. Phys. A 48 (2015) 153001 [arXiv: 1412.2296] [INSPIRE].

[76] M. Argeri et al., Magnus and Dyson Series for Master Integrals, JHEP 03 (2014) 082 [arXiv: 1401.2979] [INSPIRE].

[77] A.V. Smirnov, Algorithm FIRE - Feynman Integral REduction, JHEP 10 (2008) 107 [arXiv: 0807.3243] [INSPIRE].

[78] A.V. Smirnov and V.A. Smirnov, FIRE4, LiteRed and accompanying tools to solve integration by parts relations, Comput. Phys. Commun. 184 (2013) 2820 [arXiv:1302.5885] [inSPIRE].

[79] A.V. Smirnov, FIRE5: a C++ implementation of Feynman Integral REduction, Comput. Phys. Commun. 189 (2015) 182 [arXiv:1408.2372] [INSPIRE].

[80] G. Martinelli, C. Pittori, C.T. Sachrajda, M. Testa and A. Vladikas, A general method for nonperturbative renormalization of lattice operators, Nucl. Phys. B 445 (1995) 81 [hep-lat/9411010] [INSPIRE].

[81] A.B. Goncharov, Multiple polylogarithms, cyclotomy and modular complexes, Math. Res. Lett. 5 (1998) 497 [arXiv:1105.2076] [INSPIRE].

[82] K.-T. Chen, Iterated path integrals, Bull. Am. Math. Soc. 83 (1977) 831 [InSPIRE].

[83] E. Remiddi and J.A.M. Vermaseren, Harmonic polylogarithms, Int. J. Mod. Phys. A 15 (2000) 725 [hep-ph/9905237] [INSPIRE].

[84] J. Vollinga and S. Weinzierl, Numerical evaluation of multiple polylogarithms, Comput. Phys. Commun. 167 (2005) 177 [hep-ph/0410259] [INSPIRE].

[85] C.W. Bauer, A. Frink and R. Kreckel, Introduction to the GiNaC framework for symbolic computation within the C++ programming language, J. Symb. Comput. 33 (2002) 1 [cs/0004015] [INSPIRE].

[86] D. Maitre, HPL, a mathematica implementation of the harmonic polylogarithms, Comput. Phys. Commun. 174 (2006) 222 [hep-ph/0507152] [INSPIRE].

[87] D. Maitre, Extension of HPL to complex arguments, Comput. Phys. Commun. 183 (2012) 846 [hep-ph/0703052] [INSPIRE]. 
[88] H. Frellesvig, D. Tommasini and C. Wever, On the reduction of generalized polylogarithms to $L i_{n}$ and $L i_{2,2}$ and on the evaluation thereof, JHEP 03 (2016) 189 [arXiv:1601.02649] [INSPIRE].

[89] A.V. Smirnov, FIESTA 3: cluster-parallelizable multiloop numerical calculations in physical regions, Comput. Phys. Commun. 185 (2014) 2090 [arXiv:1312.3186] [INSPIRE].

[90] A.V. Smirnov, FIESTA4: Optimized Feynman integral calculations with GPU support, Comput. Phys. Commun. 204 (2016) 189 [arXiv:1511.03614] [INSPIRE].

[91] L.-B. Chen, W. Wang and R. Zhu, Next-to-next-to-leading order corrections to quark Quasi parton distribution functions, arXiv:2006.14825 [INSPIRE]. 